\title{
Expression of c-Myc and Beclin-1 in skin of rats after burn
}

\author{
CAIHONG LI, YUPING LIU and HONGJUAN LIU
}

\author{
Department of Burn and Plastic Surgery, Weifang People's Hospital, Weifang, Shandong 261000, P.R. China
}

Received January 15, 2018; Accepted August 1, 2018

DOI: $10.3892 /$ etm.2018.6582

\begin{abstract}
This study aimed to investigate the expression features of Beclin-1 and c-Myc in the skin burn of rats. A total of 48 Sprague-Dawley (SD) rats were randomly divided into the normal group $(n=12)$, the 3 -day burn group $(n=12)$, the 5-day burn group $(n=12)$ and the 7 -day burn group $(n=12)$. Except for the normal group, the rat models of burn were established in the other three groups, burn wounds were given routine dressing change, and rats were sacrificed at 3,5 and 7 days after modeling to collect materials. Then, immunohistochemistry was applied to detect the expression of c-Myc and Beclin-1. The expression levels of c-Myc protein and Beclin-1 protein were measured via western blotting. The expression levels of c-Myc messenger ribonucleic acid (mRNA) and Beclin-1 mRNA were detected by reverse transcription-quantitative polymerase chain reaction (RT-qPCR). In comparison with the normal group, three burn groups had significantly increased the expression of c-Myc and Beclin-1, and the differences were statistically significant $(\mathrm{P}<0.05)$. Beclin-1 expression in the 5-day burn group was obviously higher than those in the 3 and 7-day burn groups, and the differences were of statistical significance $(\mathrm{P}<0.05)$. The expression of $\mathrm{c}-\mathrm{Myc}$ in the 7-day burn group was overtly higher than those in the 3 and 5-day burn groups, and the differences showed statistical significance $(\mathrm{P}<0.05)$. The expression of Beclin-1 and c-Myc in post-burn skin tissues were gradually increased, with the Beclin-1 expression level reaching the peak on the 5th day after burn, and the expression level of c-Myc was the highest on the 7 th day after burn.
\end{abstract}

\section{Introduction}

Burn is a serious accidental injury. In particular, burn caused by fire is a challenge in surgical treatment. According to epidemiological statistics, approximately 450,000 burn patients needed treatment in the United States in 2011, on increase of approximately $340 \%$ compared with that

Correspondence to: Dr Hongjuan Liu, Department of Burn and Plastic Surgery, Weifang People's Hospital, 151 Guangwen Street, Kuiwen, Weifang, Shandong 261000, P.R. China

E-mail: zsczo894@163.com

Key words: burn, Beclin-1, c-Myc, autophagy, cell proliferation in 1995 (1,2). Therefore, how to effectively treat burns is a serious clinical challenge. The molecular mechanisms after burn are very complicated, especially the post-burn autophagy and wound healing, which are key factors related to tissue reconstruction after burn. Autophagy is an important process in vivo that is closely related to substance and energy metabolisms in cells $(3,4)$, which plays an important role in many physiological and pathological reactions and regulates various diseases and pathological reaction processes such as tumor, inflammation and cell proliferation (5). Beclin-1 is one of the hallmarks of autophagy and plays an important role in autophagy, which is considered a dynamic indicator of autophagy activity $(6,7)$. Burn wound healing involves cell proliferation, differentiation and migration, granulation tissue formation as well as extracellular matrix formation. c-Myc proto-oncogene is considered to be a mediator of cell division and plays an important role in wound healing, which can regulate tissue cell proliferation and promote cell division, thus contributing to tissue reconstruction (8). Therefore, studying the post-burn expression levels of Beclin-1 and c-Myc is of important guiding significance for the treatment of burns. Intervention can be carried out in the changes of Beclin-1 and c-Myc expression, thereby inhibiting the adverse effects of pathological responses activated due to abnormal expression on post-burn tissue reconstruction.

\section{Materials and methods}

Experimental animals and grouping. A total of 48 Sprague-Dawley (SD) rats weighing $220 \pm 20$ g (half male and half female) were purchased from Shanghai Slack Laboratory Animal Co., Ltd. (Shanghai, China) license no. SCXK 2014-0003. The above-mentioned 48 rats were randomly divided into the normal group, the 3-day burn group, the 5-day burn group and the 7-day burn group, with 12 rats in each group Rats were housed in a temperature controlled room $\left(21 \pm 2^{\circ} \mathrm{C}\right)$ on a 12 -h light/dark cycle (lights on at 06:00). All rats had free access to water and food.

This study was approved by the Animal Ethics Committee of Weifang People's Hospital Animal Center (Weifang, China).

Experimental reagents. Main reagents used in this study included primary antibodies anti-Beclin-1 and anti-c-Myc, immunohistochemical kits (KIT-9710; Maxim, San Jose, CA, USA), AceQ reverse transcription-quantitative polymerase chain reaction (RT-qPCR) SYBR-Green Master mix kits (Q111-02/03) and HiScript II Q RT Sperfect for 
Table I. Primer sequences.

\begin{tabular}{lll}
\hline Gene name & & \multicolumn{1}{c}{ Primer sequences } \\
\hline Beclin-1 & Upstream: & 5'-CGGAATTCTATGGAAGGGTCTAAGACGTCC-3' \\
& Downstream: & 5'-CGGGATCCTCATTGTTATAAAATTGTGAGGACA-3' \\
$c-M y c$ & Upstream: & 5'-ATCACAGCCCTCACTCAC-3' \\
& Downstream: & 5'-ACAGATTCCACAAGGTGC-3' \\
GADPH & Upstream: & 5'-ACGGCAAGTTCAACGGCACAG-3' \\
& Downstream: & 5'-GAAGACGCCAGTAGACTCCACGAC-3' \\
\hline
\end{tabular}

qPCR [(+genomic deoxyribonucleic acid (gDNA) wiper] kits (R223-01) (both from Vazyme Biotech Co., Ltd., Nanjing, China).

Experimental equipments. Main experimental equipment used in this study were an optical microscope (Leica DMI 4000B/DFC425C), a fluorescence RT-qPCR instrument (ABI 7500), Image-lab image analysis system, Image-Pro image analysis system (both from Bio-Rad Laboratories, Inc., Hercules, CA, USA) and a digital thermostatic water bath kettle (Changzhou Guohua Electric Appliance Co., Ltd., Changzhou, China).

Establishment of burn models. Rats were intraperitoneally injected with 7\% chloral hydrate, and the injection volume was $5 \mathrm{ml} / \mathrm{kg}$. After successful anesthesia, hair on the back of the rats was removed to expose the skin. After disinfection, the back of the rats was immersed in the digital thermostat water bath kettle for $10 \mathrm{sec}$, resulting in burn wounds.

Treatment in each group. Burn models were prepared in the 3 , 5 and 7-day burn groups according to the above establishment method for burn models. After successful modeling, wounds were given routine dressing change and packing. Then, rats were sacrificed at 3,5 and 7 days after modeling, respectively, and skin tissues of burn wounds on the back were collected for experiments. However, rats in the normal group received no treatment and were sacrificed directly to collect normal skin tissues on the back for experiments.

Material collection. After successful anesthesia, 6 rats in each group were fixed with paraformaldehyde, and skin tissues on the back (with an area of $\sim 1 \mathrm{~cm}^{2}$ ) were collected and placed in $4 \%$ paraformaldehyde, followed by fixation at $4^{\circ} \mathrm{C}$ for $48 \mathrm{~h}$. Then, paraffin tissue sections were made for immunohistochemical detection. Skin tissues on the back (with an area of $\sim 1 \mathrm{~cm}^{2}$ ) were directly taken from the remaining 6 rats and placed in epoxy resin (EP) tubes for western blotting detection.

Immunohistochemistry. Paraffin tissue sections $(5 \mu \mathrm{m})$ were conventionally dewaxed, hydrated, added with citric acid buffer, and heated in a microwave oven for antigen retrieval. Then, sections were rinsed with phosphate-buffer saline (PBS) solution and added with endogenous peroxidase blocking agent for $10 \mathrm{~min}$ of incubation. After that, sections were rinsed with PBS solution and added with goat serum for 20 min of blocking. Then, serum-blocking solution was removed, and
anti-Beclin-1 primary antibody (1:200; cat. no. ab62557) and anti-c-Myc primary antibody (1:200; cat. no. ab39688), both from Abcam, Cambridge, MA, USA, were added for incubation overnight at $4^{\circ} \mathrm{C}$. After that, sections were rinsed with PBS solution and incubated with secondary goat anti-rabbit (HRP) IgG antibody (dilution, 1:500; cat. no. ab6721; Abcam) for $10 \mathrm{~min}$. Then, PBS solution was used for rinsing, and streptavidin peroxidase was added for $10 \mathrm{~min}$ of incubation. Last, sections were subjected to color development via diaminobenzidine (DAB), counterstained with hematoxylin, mounted with neutral balsam, observed under the microscope (Leica) and photographed.

Western blotting. Skin tissues stored at $-20^{\circ} \mathrm{C}$ for standby application were added with lysis solution, followed by ice bath for $60 \mathrm{~min}$. Then, tissues were centrifuged at $14,000 \mathrm{x} \mathrm{g}$ for $10 \mathrm{~min}$, and protein was quantified by bicinchoninic acid (BCA) assay. The standard curve and optical density were measured via a microplate reader (Bio-Rad) and the concentration of protein was calculated. After protein was denatured, samples were separated by sodium dodecyl sulfate-polyacrylamide gel electrophoresis (SDS-PAGE) with corresponding concentration. When marker protein reached to the bottom of the glass plate, and sample protein was almost in a straight line at the bottom, gel running was stopped. Then, the sample was transferred onto a polyvinylidene fluoride (PVDF) membrane for blocking, followed by washing with tetrapropyl benzene sulfonate (TPBS) 3 times. After that, membrane was blocked with blocking solution for $1.5 \mathrm{~h}$, added with anti-Beclin-1 primary antibody $(1: 1,000)$ and anti-c-Myc primary antibody $(1: 1,000)$, rinsed with Tris-buffered saline with Tween-20 (TBST), and added with secondary goat anti-rabbit (HRP) IgG antibody (dilution, 1:1,000; cat. no. ab6721; Abcam), USA), followed by rinsing with TBST. After the secondary antibody was removed by TBST washing, development was started. Finally, a membrane was placed in a chemiluminescence reagent for $1 \mathrm{~min}$ of reaction, developed in the dark and analyzed using the gel scanning imaging system.

RT-qPCR detection. Total ribonucleic acid (RNA) was extracted from spare bone stored at $-20^{\circ} \mathrm{C}$ using RNA extraction kits. The extracted total RNA was reverse-transcribed into complementary deoxyribonucleic acid (cDNA) through reverse transcription kits, and the volume of the reaction system was $20 \mu \mathrm{l}$. Reaction conditions: reaction at $51^{\circ} \mathrm{C}$ for $2 \mathrm{~min}$, predenaturation at $96^{\circ} \mathrm{C}$ for $10 \mathrm{~min}$, denaturation at $96^{\circ} \mathrm{C}$ for $10 \mathrm{sec}$, annealing at $60^{\circ} \mathrm{C}$ for $30 \mathrm{sec}$, for 40 cycles. 


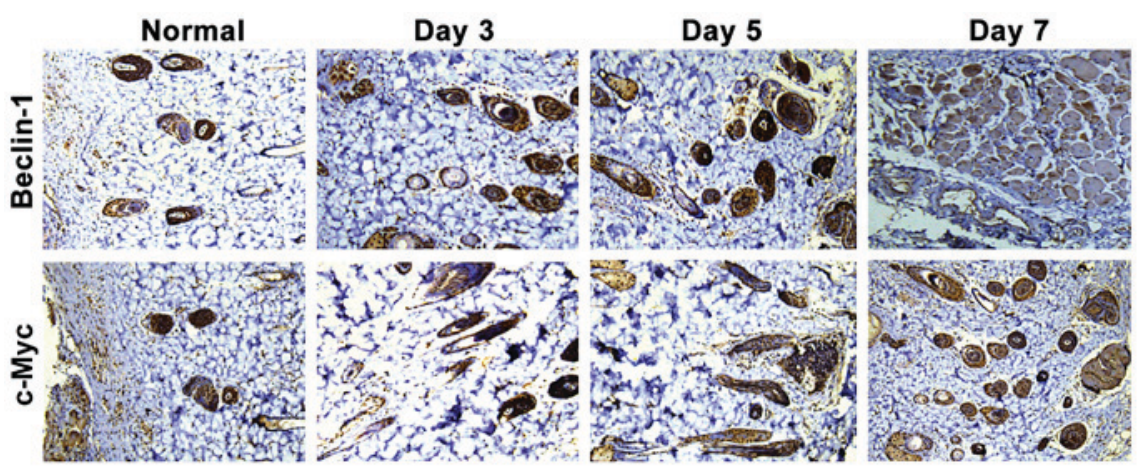

Figure 1. Expression levels of Beclin-1 and c-Myc detected via immunohistochemistry.

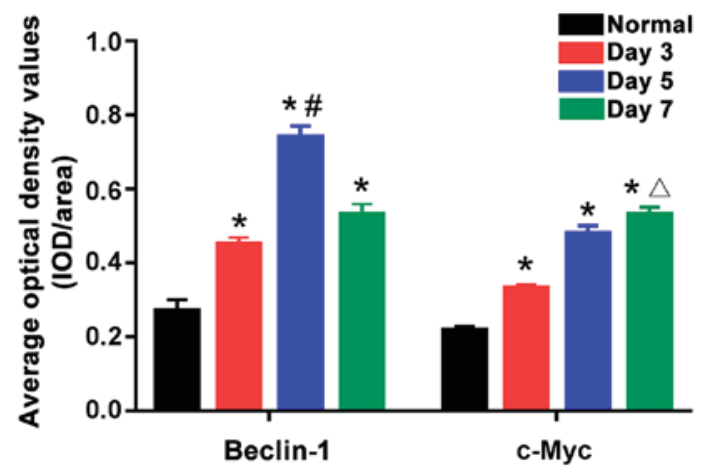

Figure 2. Average optical density values of Beclin-1 and c-Myc. ${ }^{*} \mathrm{P}<0.05$, in comparison with the normal group; ${ }^{~} \mathrm{P}<0.05$, in comparison with the 3 and 7-day burn groups; ${ }^{\wedge} \mathrm{P}<0.05$, in comparison with the 3 - and 5 -day burn groups.

A

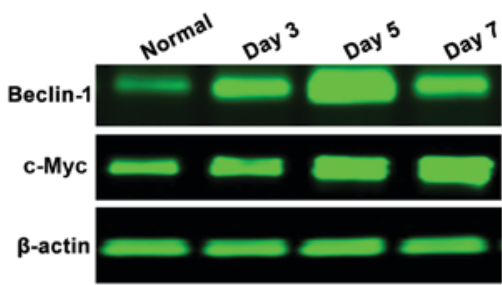

B

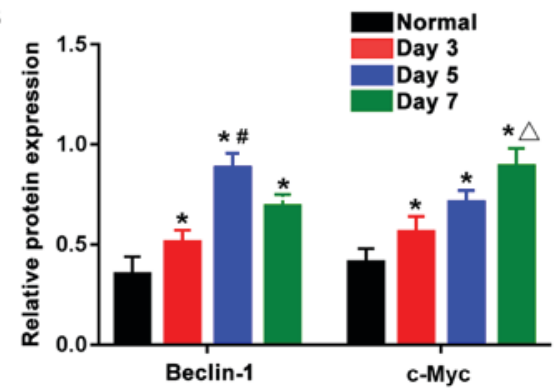

Figure 3. Protein expression levels of Beclin-1 and c-Myc in each group. (A) Expression levels of Beclin-1 protein and c-Myc protein detected by western blotting. (B) ${ }^{*} \mathrm{P}<0.05$, in comparison with the normal group; ${ }^{\prime \prime} \mathrm{P}<0.05$, in comparison with the 3 - and 7 -day burn groups; ${ }^{\triangle} \mathrm{P}<0.05$, in comparison with the 3- and 5-day burn groups.

Glyceraldehyde-3-phosphate dehydrogenase (GAPDH) was used as an internal control, and relative messenger RNA (mRNA) expression levels of Beclin-1 and c-Myc were calculated. Primer sequences are shown in Table I.

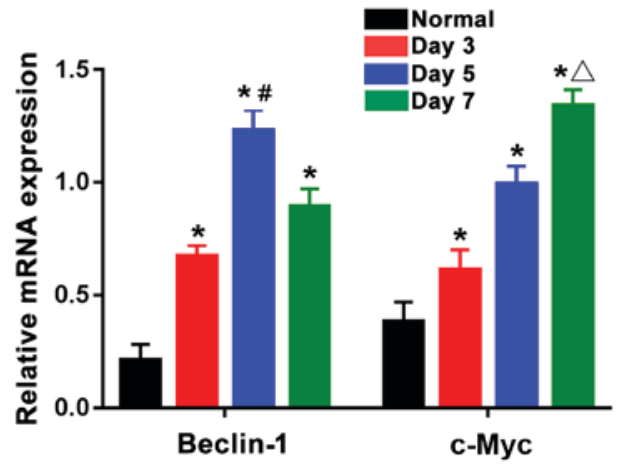

Figure 4. mRNA expression levels of Beclin-1 and c-Myc. ${ }^{*} \mathrm{P}<0.05$, in comparison with the normal group; ${ }^{\#} \mathrm{P}<0.05$, in comparison with the 3 - and 7 -day burn groups; ${ }^{\wedge} \mathrm{P}<0.05$, in comparison with the 3- and 5-day burn groups.

Observation indexes. The expression levels of Beclin-1 and c-Myc in skin tissues were detected by immunohistochemistry. The protein expression levels of Beclin-1 and c-Myc in skin tissues were detected through western blotting. The mRNA expression levels of Beclin-1 and c-Myc in skin tissues were detected via RT-qPCR.

Statistical analysis. Statistical Product and Service Solutions (SPSS) 20.0 software (IBM SPSS, Armonk, NY, USA) was used for statistical analysis in this study. Measurement data were expressed as mean \pm standard deviation. Comparison between multiple groups was done using One-way ANOVA test followed by post hoc test (Least Significant Difference). The Chi-square test was used for enumeration data.

\section{Results}

Expression levels of Beclin-1 and c-Myc detected via immunohistochemistry. As shown in Fig. 1, the positive expression of Beclin-1 and c-Myc were tan color. The positive expression of Beclin-1 and c-Myc in the normal group were fewer, while those in the three burn groups were significantly increased, which showed statistically significant differences $(\mathrm{P}<0.05)$. According to statistical analysis of positive expression (Fig. 2), the 5-day burn group had the highest positive expression level of Beclin-1, which was obviously higher than those in the 3- and 7-day burn groups, showing statistically significant differences $(\mathrm{P}<0.05)$. In addition, the positive expression level of c-Myc in the 7-day burn group was 
the highest, which was evidently higher than those in the 3-and 5-day burn groups, showing statistically significant differences $(\mathrm{P}<0.05)$. This suggests that after burn, the expression levels of Beclin-1 and c-Myc in skin tissues are gradually increased with the expression level of Beclin-1 reaching the peak around the 5 th day after burn and then beginning to gradually decline, and that of c-Myc was the highest around the 7th day after burn.

Protein expression levels of Beclin-1 and $c$-Myc detected by western blotting. As shown in Fig. 3A, the expression levels of Beclin-1 protein and c-Myc protein were reduced in the normal group, while those in the three burn groups were overtly increased, and the differences were statistically significant $(\mathrm{P}<0.05)$. The expression levels of Beclin-1 and c-Myc proteins were subjected to statistical analysis, and the results (Fig. 3B) showed that the Beclin-1 protein expression level in the 5-day burn group was the highest, which was significantly greater than those in the 3-and 7-day burn groups, showing statistically significant differences $(\mathrm{P}<0.05)$. The expression level of c-Myc protein in the 7-day burn group was the highest and significantly higher than those in the 3- and 5-day burn groups. This indicates that the expression levels of Beclin-1 and c-Myc proteins in skin tissues are gradually increased after burn with the Beclin-1 protein expression level reaching the peak around the 5th day after burn and then beginning to decline, and the c-Myc protein expression level was the highest around the 7th day after burn.

Expression levels of Beclin-1 mRNA and c-Myc mRNA detected by RT-qPCR. According to Fig. 4, the mRNA expression levels of Beclin-1 and c-Myc were lower in the normal group, while those in the three burn groups were distinctly elevated, showing statistically significant differences $(\mathrm{P}<0.05)$. The mRNA expression of Beclin-1 was the highest in the 5-day burn group, which was clearly higher than those in the 3- and 7-day burn groups, and the differences were statistically significant $(\mathrm{P}<0.05)$. The mRNA expression of c-Myc in the 7-day burn group was the highest and obviously higher than those in the 3- and 5-day burn groups. This suggests that the expression of Beclin-1 and c-Myc mRNAs in skin tissues are gradually increased after burn with the mRNA expression level of Beclin-1 reaching the peak around the 5th day after burn and then beginning to gradually decline, and that of c-Myc was the highest around the 7th day after burn.

\section{Discussion}

Autophagy and cell proliferation in skin tissues after burn are important factors affecting wound healing. Autophagy is closely related to recycling and utilization of intracellular macromolecular substances, removal of damaged tissues and maintenance of the stability of intracellular environment $(9,10)$. Present studies have suggested that there is a close relationship between autophagy and apoptosis. Although their characteristics and pathological mechanisms are different, autophagy and apoptosis are not separate from each other, have many common stimulating factors and regulatory proteins, and are interrelated and complex (11). The process of autophagy exists in physiological and pathological processes, such as injury and disease. In the pathological processes of injury and disease, intervention in autophagy can have a serious impact on cell recovery process $(12,13)$. Currently, studies have indicated that as a mammalian homologue of the yeast autophagy-related protein 6 (ATG6)/vacuolar protein sorting-associated protein 30 (Vps30) gene, Beclin-1 binds to its ligand and regulates the activity of autophagy, thus playing an important role in autophagy (14). Further studies have found that different domains of Beclin-1 bind to autophagy regulatory proteins to form protein complexes, thus regulating autophagy $(15,16)$. This study showed that Beclin-1 expression is gradually increased in skin tissues after burn, thereby regulating the process of autophagy started in the cells. The level of autophagy is getting higher and higher. The Beclin-1 expression reaches a peak on the 5th day after burn, and the autophagy level in cells also reaches the peak, leading to autophagic cell death. Then, the expression level is gradually decreased, and the level of autophagy begins to recede.

Cell proliferation is also an important factor affecting wound healing after burn. c-Myc is an important oncogene that regulates cell division and proliferation. In addition, c-Myc is an important mediator of mitosis, which can prompt cells in the quiescent stage to rapidly transfer into the division stage, thus promoting cell division and proliferation (17). Meanwhile, c-Myc is a downstream substrate for various cell signaling pathways, which can act as a transcription factor and transmit information in the nucleus so as to regulate cell cycle, promote cell proliferation and block cell differentiation (18). A study suggested that c-Myc cannot only promote E2F to bind to corresponding DNA by activating cyclin E/cyclin-dependent kinase 2 (CDK2) so as to mediate cell proliferation, but also regulate cell cycle by regulating transferrin receptor-1 (TFRC1) so as to regulate cell proliferation (19). At the same time, c-Myc does not always lead to cell proliferation. When the peripheral environment where cells are located allows the proliferation of cells, c-Myc acts on the downstream signaling pathways, releases the signal of growth and secretes a variety of growth-related factors, so as to maintain cell proliferation. When the signal promoting cell growth disappears, the overexpression of c-Myc causes apoptosis, thus allowing cells to maintain homeostasis of reproduction and apoptosis (20). This study showed that the c-Myc expression begins to increase after burn, indicating that local injured skin tissue cells begin to proliferate and repair, and c-Myc expression on the 7th day after burn is significantly increased. This indicates that skin tissue cells have rapid proliferation, and damaged skin tissues start a process of rapid repair. At the same time, conside ring the expression regularity of Beclin-1, it was believed that autophagy occurs in cells when the Beclin-1 expression is increased in the early stage of the injury, causing a large number of cell death, and autophagy reaches the highest level when the Beclin-1 expression reaches a peak on the 5th day after burn, which is not conducive to cell proliferation, so it was observed that the c-Myc expression levels on the 3rd and 5th day after burn were not high, indicating that the c-Myc expression is inhibited by autophagy caused by the high expression of Beclin-1. On the 7th day after burn, the expression of Beclin-1 is significantly decreased, and the level of autophagy is clearly decreased. Therefore, the inhibition effect of autophagy on c-Myc is weakened. At the same time, 
the environment where cells are located is favorable for the proliferation of cells. Therefore, the expression of c-Myc is increased, and massive cells begin to proliferate, promoting local wound repair.

In conclusion, the expression of Beclin-1 and c-Myc after burn has certain regularities, which can be used as effective targets for the treatment of burns. At the same time, corresponding intervention can be carried out for the expression features of Beclin-1 and c-Myc after burn, namely, inhibit the expression of Beclin-1 after burn to inhibit autophagy, and promote the expression of c-Myc to benefit cell proliferation, thereby promoting wound repair and healing.

\section{Acknowledgements}

Not applicable.

\section{Funding}

No funding was received.

\section{Availability of data and materials}

All data generated or analyzed during this study are included in this published article.

\section{Authors' contributions}

CL analyzed and HL interpreted the data. HL performed experiments. CL wrote the manuscript. YL carried out the statistical analysis and CL edited the language. All authors have read and approved the final study.

\section{Ethics approval and consent to participate}

This study was approved by the Animal Ethics Committee of Weifang People's Hospital Animal Center (Weifang, China).

\section{Patient consent for publication}

Not applicable.

\section{Competing interests}

The authors declare that they have no competing interests.

\section{References}

1. YoshimuraK, SugaH and EtoH: Adipose-derived stem/progenitor cells: Roles in adipose tissue remodeling and potential use for soft tissue augmentation. Regen Med 4: 265-273, 2009.
2. Tomita K, Madura T, Sakai Y, Yano K, Terenghi G and Hosokawa K: Glial differentiation of human adipose-derived stem cells: Implications for cell-based transplantation therapy. Neuroscience 236: 55-65, 2013.

3. Tanida I: Autophagy basics. Microbiol Immunol 55: 1-11, 2011.

4. Czaja MJ, Ding WX, Donohue TM Jr, Friedman SL, Kim JS, Komatsu M, Lemasters JJ, Lemoine A, Lin JD, Ou JH, et al: Functions of autophagy in normal and diseased liver. Autophagy 9: 1131-1158, 2013.

5. Doria A, Gatto M and Punzi L: Autophagy in human health and disease. N Engl J Med 368: 1845-1846, 2013.

6. Wang W, Fan H, Zhou Y, Duan P, Zhao G and Wu G: Knockdown of autophagy-related gene BECLIN1 promotes cell growth and inhibits apoptosis in the A549 human lung cancer cell line. Mol Med Rep 7: 1501-1505, 2013.

7. Ruck A, Attonito J, Garces KT, Núnez L, Palmisano NJ, Rubel Z, Bai Z, Nguyen KC, Sun L, Grant BD, et al: The Atg6/Vps30/Beclin 1 ortholog BEC-1 mediates endocytic retrograde transport in addition to autophagy in $\mathrm{C}$. elegans. Autophagy 7: 386-400, 2011.

8. Clark DE, Li C, Wang W, Martin SK and Suttie JM: Vascular localization and proliferation in the growing tip of the deer antler. Anat Rec A Discov Mol Cell Evol Biol 288: 973-981, 2006.

9. Tanida I: Autophagosome formation and molecular mechanism of autophagy. Antioxid Redox Signal 14: 2201-2214, 2011.

10. Goehe RW, Bristol ML, Wilson EN and Gewirtz DA: Autophagy, senescence, and apoptosis. Methods Mol Biol 962: 31-48, 2013.

11. Eisenberg-Lerner A, Bialik S, Simon HU and Kimchi A: Life and death partners: Apoptosis, autophagy and the cross-talk between them. Cell Death Differ 16: 966-975, 2009.

12. Sun Y, Liu JH, Sui YX, Jin L, Yang Y, Lin SM and Shi H: Beclin1 overexpression inhibitis proliferation, invasion and migration of CaSki cervical cancer cells. Asian Pac J Cancer Prev 12: 1269-1273, 2011

13. Mijaljica D, Prescott M and Devenish RJ: Autophagy in disease. Methods Mol Biol 648: 79-92, 2010.

14. Matsunaga K, Saitoh T, Tabata K, Omori H, Satoh T, Kurotori N, Maejima I, Shirahama-Noda K, Ichimura T, Isobe T, et al: Two Beclin 1-binding proteins, Atg14L and Rubicon, reciprocally regulate autophagy at different stages. Nat Cell Biol 11: 385-396, 2009.

15. Wirawan E, Lippens S, Vanden Berghe T, Romagnoli A, Fimia GM, Piacentini M and Vandenabeele P: Beclin1: A role in membrane dynamics and beyond. Autophagy 8: 6-17, 2012.

16. Sahni S, Merlot AM, Krishan S, Jansson PJ and Richardson DR: Gene of the month: BECN1. J Clin Pathol 67: 656-660, 2014.

17. Florin L, Hummerich L, Dittrich BT, Kokocinski F, Wrobel G, Gack S, Schorpp-Kistner M, Werner S, Hahn M, Lichter P, et al: Identification of novel AP-1 target genes in fibroblasts regulated during cutaneous wound healing. Oncogene 23: 7005-7017, 2004.

18. Cui J, Waltman P, Le VH and Lewis EA: The effect of molecular crowding on the stability of human c-MYC promoter sequence I-motif at neutral pH. Molecules 18: 12751-12767, 2013.

19. Kalra N and Kumar V: c-Fos is a mediator of the c-myc-induced apoptotic signaling in serum-deprived hepatoma cells via the p38 mitogen-activated protein kinase pathway. J Biol Chem 279: 25313-25319, 2004.

20. DuHadaway JB, Sakamuro D, Ewert DL and Prendergast GC: Bin1 mediates apoptosis by c-Myc in transformed primary cells. Cancer Res 61: 3151-3156, 2001. 